\title{
Alltagspraktiken des Erzählens
}

\author{
Constanze Spieß • Doris Tophinke
}

Online publiziert: 20. März 2018

(C) Springer-Verlag GmbH Deutschland, ein Teil von Springer Nature 2018

Erzählen findet sich keineswegs nur in ästhetisch-fiktionalen Domänen, sondern ist in mehr oder weniger ausgebauter Form in allen Bereichen des Alltags präsent. Erzählen als Alltagspraktik ist vielfach eingelassen in andere kulturelle Praktiken. Es begegnet als konversationelles Erzählen wohl in beinahe jeder alltäglichen Faceto-face-Interaktion, da in der Interaktion immer auch Erlebnisse und Erfahrungen narrativ vergegenwärtigt und bearbeitet werden. So konstatiert Bal: »There is hardly a cultural practice where some form and degree of narrativity does not play a part.« (Bal 2007, S. 251)

Das Erzählen als Alltagspraktik und Kulturtechnik ist für viele wissenschaftliche Disziplinen interessant, die - wie der Sammelband von Martínez (2017) zeigt - jeweils spezifische theoretisch-methodische Zugänge entwickelt haben. Zu den führenden Disziplinen gehört seit vielen Jahren die Linguistik. Gegenwärtig wird auf das »Gründungsdokument linguistischer Erzählforschung « (Kern/Morek/Ohlhus 2012, S. 1) von Labov/Waletzky (1967) im Kontext linguistischer Erzählforschung erneut kritisch und modifizierend Bezug genommen. Nach Labov/Waletzky (1967) lassen sich mündliche Erzählungen in funktionale Abschnitte - die Orientierung, Komplikation, Evaluation, Resolution und Koda - gliedern, die jeweils bestimmte »Fragen« bearbeiten und deren Abfolge einem festen Muster folgt. Ihr Modell wurde vielfach aufgegriffen, im Hinblick auf die Annahme eines festen Sequenzmusters aber auch stark kritisiert. Aus der Auseinandersetzung mit Labov/Waletzky (1967)

\footnotetext{
C. Spieß $(\bowtie)$

Institut für Germanistik, Karl-Franzens-Universität Graz, Graz, Österreich

E-Mail: constanze.spiess@uni-graz.at

D. Tophinke

Institut für Germanistik und Vergleichende Literaturwissenschaft, Universität Paderborn, Paderborn, Deutschland

E-Mail: doris.tophinke@upb.de
} 
resultieren viele Ansätze, die sich mit der Grundstruktur von Erzählungen nicht nur in monologischer Kommunikation, sondern v. a. auch im Hinblick auf interaktionale, dialogische Kommunikation befassen (vgl. dazu auch Kotthoff in diesem Band).

Dass alltägliches Erzählen funktional eingebunden ist in interaktionale Kontexte, wurde v. a. in der interaktionalen Linguistik und in der Gesprächsforschung thematisiert (vgl. hier beispielsweise Günthner 1999 oder 2012, Kotthoff 2011 oder 2015; vgl. dazu auch die Beiträge von Hoffmann, Kotthoff und König/Oloff sowie Quasthoff/Stude in diesem Band). So sind Erzählungen in alltagssprachlichen Zusammenhängen beteiligt an der Herstellung scheinbar >objektiver< Tatsachen unserer Lebenswirklichkeiten. Sie konstruieren Wirklichkeit(en) kontextgebunden (Luckmann 1988, Berger/Luckmann 2004), dienen der Konstruktion individueller und sozialer Identität, markieren etwa die Zugehörigkeit zu sozialen Gruppen oder erzeugen Ausgrenzungen (vgl. Günthner 2012). Zugleich werden durch Narrationen in alltagssprachlichen Zusammenhängen soziale Situationen interaktiv hervorgebracht. In seiner sprachlichen Typik, seiner sozialen Funktionalität und Sinnhaftigkeit, seiner Bindung an alltägliche Interaktionskonstellationen (z. B. Small Talk, Tischgespräch, ...) und nicht zuletzt in seiner Erlernbarkeit und Routinisierung lässt sich das Erzählen auch praxistheoretisch perspektivieren (vgl. De Fina und Georgakopoulou 2015).

Linguistische Forschungen zum Erzählen sind in der Spracherwerbsforschung, der Gesprächslinguistik, der interaktionalen Linguistik oder auch der Textlinguistik verortet. Sie widmen sich unterschiedlichen Aspekten des Erzählens und sind durch unterschiedliche theoretisch-methodische Zugänge bestimmt. Im Vordergrund steht - vereinfacht gesprochen - entweder das Erzählen als dynamischer (interaktionaler) Prozess oder der Text als Produkt des Erzählens. Während interaktionale Ansätze die interaktive Herstellung sozialer Tatsachen durch kommunikative Verfahren des Erzählens untersuchen und dabei sequenzielle Muster, grammatische Besonderheiten und deren Funktionalität im Kontext der Interaktion sowie deren lokale Einbindung und den Aspekt der Ko-Konstruktion von Erzählungen durch alle an der Interaktion beteiligten Personen hervorheben, werden in textlinguistischen Ansätzen in erster Linie die Spezifik narrativer Textualität sowie die semantisch-konzeptionellen Eigenschaften von Erzähltexten betrachtet. Text- und genrebezogene Ansätze gehen hier im Anschluss an Labov/Waletzky (1967) von narrativen Themenentfaltungsstrukturen aus. Im Kontext der interaktionalen Erzählforschung sind eine ganze Reihe von Arbeiten entstanden, die die Spezifik der interaktional hervorgebrachten Erzählschemata in verschiedenen mehr oder weniger institutionell geprägten Kommunikationsbereichen beschreiben. $\mathrm{Zu}$ nennen wären hier beispielsweise Untersuchungen zur Arzt-Patientenkommunikation (vgl. Birkner 2017), zur Kommunikation in Schule und anderen Bildungseinrichtungen (z. B. die Analyse von Sprechstundengesprächen bzw. Elternsprechtagsgesprächen, vgl. hierzu Kotthoff 2015), Arbeiten zu autobiographischem Alltagserzählen in narrativen Interviews (vgl. dazu u.a. Leonardi/Thüne/Betten 2016), Arbeiten, die die Entwicklung und Förderung von Erzählfähigkeit in den Blick nehmen (vgl. hier Becker, 2009, 2011, Becker/Stude 2017, Quasthoff 1980, 2001) oder die sich mit sprachlichen Komponenten/Mustern des Erzählens im Kontext literarischer Texte aus einer linguistischen Perspektive auseinandersetzen (vgl. Fix 2007 und 2015). 
Abb. 1 Ebenen und Beschreibungsdimensionen im Kontext des Erzählens und von Erzählungen

\begin{tabular}{l}
\hline sprachliche Ebenen \\
\hline phonologische Ebene \\
morphologische Ebene \\
syntaktische Ebene \\
textuelle Ebene \\
sequenzielle Ebene \\
diskursive Ebene \\
\hline
\end{tabular}

Beschreibungsdimensionen

formal-grammatische

Dimension

semantisch-konzeptuelle

Dimension

sozio-pragmatische Dimension

In den Untersuchungen zum Erzählen werden verschiedene sprachliche Ebenen - von der phonologischen Ebene bis hin zur diskursiven Ebene (s. Abb. 1) - und Aspekte in den Blick genommen, wobei sich das Interesse auf formal-grammatische, semantisch-konzeptuelle und/oder sozio-pragmatische Aspekte richten kann (die Untersuchung sich demzufolge auch auf die entsprechende Beschreibungsdimension bezieht). Erzählen wird dabei als Verfahren erkennbar, das nicht Selbstzweck ist, sondern das - in einfacher oder entfalteter Form - in der sprachlichen Interaktion bzw. in sozialen Praktiken funktionalisiert ist und dessen Sinn sich auch nur im Kontext erschließt. Schon Schegloff (1997) hat auf diese funktionale Bindung des Erzählens hingewiesen:

For the most part, people tell stories to do something - to complain, to boast, to inform, to alert, to tease, to explain, to excuse or justify, or to provide for an interactional environment in whose course or context or interstices such actions and interactional inflections can be accomplished. (Schegloff 1997, S. 97)

Auf einer abstrakteren Ebene können die Funktionen des alltäglichen Erzählens folgendermaßen zusammenfassend beschrieben werden:

- Erzählen hat eine identitätsstiftende oder gar identitätskonstruierende Funktion (z. B. beim autobiographischen Erzählen, vgl. hierzu Leonardi/Thüne/Betten 2016, Papenbrock/Tophinke 2016, Tophinke 2002).

- Durch Erzählen werden Zugehörigkeiten zu sozialen Gruppen und Kulturen hervorgebracht oder indiziert (vgl. Mandelbaum 2003, Günthner 2012).

- Erzählen kann zugleich eine abgrenzende und eine integrierende Funktion im Kontext der Markierung von Zugehörigkeit oder Nicht-Zugehörigkeit zu sozialen Gruppen haben (vgl. Günthner 2012, Becker/Stude 2017).

- Durch Erzählen ist soziale Positionierung möglich (vgl. Günthner 2005).

- Erzählen dient der Herstellung von sozialer Nähe oder Distanz und hat somit eine wichtige Funktion im Kontext sozialer Beziehungen.

- Erzählen ist relevant für die Wissensaneignung, -verarbeitung, -organisation und -konstitution (Birkner 2017).

- Das Erzählen im interaktionalen Kontext dient der Vergewisserung über eine geteilte Welt- und Wirklichkeitssicht (Norrick 2007).

- Durch Erzählen teilen wir kommunikativ soziale Welten (Kern/Morek/Ohlhus 2012, Rehbein 2012). 
Diese Liste der Funktionen ist nicht vollständig. Es gibt sicher noch viele weitere Funktionen, die hier nicht alle genannt werden sollen. Eine genauere Bestimmung der Funktion ist ohnehin nur im Rahmen empirischer Analysen spezifischer Erzählsituationen bzw. Erzählpraktiken möglich.

\section{Neuere Entwicklungen}

Während sich die Erzählforschung in der Vergangenheit Narrationen und narrativen Elementen in der Alltagsinteraktion, in narrativen Interviews, in geschriebener Sprache (hier u. a. in Gebrauchstexten und in fiktionaler Literatur) gewidmet hat, nehmen neuere Forschungen stärker auch Formen und Kontexte des Erzählens in den digitalen Medien in den Blick. Das Interesse richtet sich auf die Spezifik des Erzählens in den digitalen Medien, etwa auch darauf, wie die technischen Ressourcen für das Erzählen genutzt werden und wie (interaktionale) Erzählpraktiken bzw. -traditionen, die außerhalb der digitalen Medien entstanden, in die digitalen Medien transponiert werden (vgl. hierzu etwa Tophinke 2009, 2017).

Das vorliegende Themenheft spiegelt das breite Spektrum an linguistischen $\mathrm{Zu}$ gängen zum Erzählen. Die versammelten Beiträge bieten einmal einen vertieften Einblick in zentrale Ergebnisse der linguistischen Erzählforschung. Zum anderen präsentiert das Themenheft - auf den bereits etablierten Ansätzen linguistischer Erzählforschung aufbauend - Beiträge, die methodischen oder theoretischen Ansätzen folgen, die in der Analyse des Erzählens noch relativ neu sind. So wird etwa gezeigt, welche Möglichkeiten sich durch den Einsatz korpuslinguistischer Verfahren für die Analyse narrativer Muster in Diskursen ergeben und wie sich innerhalb großer Textkorpora narrative Muster und Routinen berechnen lassen, und es werden Formen des Erzählens in den digitalen Medien betrachtet (vgl. die Beiträge von Bubenhofer, Gredel/Mell, Weidacher in diesem Band).

Die Rolle körperlicher und verkörperlichter Praktiken, die in der Multimodalitätsforschung seit Jahren Gegenstand von Untersuchungen sind, wird im Zusammenhang des Alltagserzählens im Zusammenspiel mit sprachlichen Äußerungen untersucht (vgl. König/Oloff in diesem Band). Narrative Texte werden einer konstruktionsgrammatischen Analyse unterzogen, bei der gezeigt wird, dass es spezifische Konstruktionen gibt, die Narrationen konstituieren (vgl. Ziem/Lasch in diesem Band). Der in anderen Disziplinen populäre Terminus des Narrativs spielt in der linguistischen Erzählforschung erst seit kurzer Zeit eine Rolle, und zwar im Kontext diskursanalytischer Untersuchungen. Bubenhofer et al. (2013) definieren Narrative als »sozial akzeptierte Interpretationsmuster, die unsere Wahrnehmung und Darstellung von Zusammenhängen ermöglichen und gleichzeitig begrenzen. Obwohl sie eine wichtige Funktion bei der Konstruktion der Grenzen des Sagbaren im Foucault'schen Sinn haben, wurden sie von der linguistischen Diskursanalyse bislang weitgehend vernachlässigt.« Im Beitrag von Bubenhofer im vorliegenden Themenheft ist diese Definition leitend für die korpuslinguistische Analyse. 
Die hier versammelten Beiträge spiegeln nur Ausschnitte der aktuellen linguistischen Erzählforschung. Es sollte aber deutlich werden, aus welch unterschiedlichen Teilbereichen und linguistischen Perspektiven dem Alltagsphänomen des Erzählens und alltagssprachlichen Erzählungen nachgegangen werden kann.

\section{Zu den Beiträgen im Einzelnen}

Beiträge, die sich mit einer interaktionalen Perspektive auf Face-to-Face-Kommunikation konzentrieren und vor allem die gemeinschaftlichen Herstellungen alltäglicher Erzählungen in den Blick nehmen, sind in einem ersten Teil des Heftes versammelt. Es handelt sich um die Beiträge von Helga Kotthoff, Ludger Hoffmann, Uta Quasthoff und Juliane Stude sowie von Katharina König und Florence Oloff, die je verschiedene Aspekte fokussieren und aus unterschiedlichen Perspektiven die Etablierung von Alltagserzählungen betrachten.

Aus funktionalpragmatischer Perspektive nimmt Ludger Hoffmann mit seinem Beitrag »Erzählen aus funktional-pragmatischer Perspektive« das alltägliche, nichtinstitutionelle Erzählen in den Blick. Erzählen fasst er als Diskursmuster auf, das einem bestimmten Strukturmuster folgt und das als mentale und interaktionale Handlungsstruktur aufzufassen ist, die gesellschaftliche Zwecke erfüllt. Diskursmuster können mehr oder weniger flexibel sein, sie sind gekennzeichnet durch Handlungsverkettungen, die entweder ohne Sprecherwechsel ablaufen oder aber durch Sprecherwechsel sequenziell alternierend strukturiert sind. Am Beispiel einer Alltagserzählung arbeitet Hoffmann systematisch die einzelnen Strukturelemente der konkreten Erzählung heraus. Anhand der Analyse wird deutlich, wie durch die an der Narration beteiligten Personen eine gemeinsame kommunikative Welt hergestellt wird.

Helga Kotthoff geht in ihrem Beitrag »Nicht ausgebaute Erzählungen« auf die interaktionale Herstellung von im Alltag geführten Gesprächen ein, die u. a. fragmentarische Narrationen enthalten. Die Funktion fragmentarischen Erzählens steht dabei zumeist im Dienst übergeordneter Zwecke des Gesprächs. Ausgehend von einem Überblick sowohl über die prozessorientierte, konversationsanalytische Erzählforschung, die die gemeinschaftliche Herstellung von Erzählungen fokussiert, als auch über die produktorientierte Erzählforschung (Labov/Waletzky), bei denen die ForscherInnen davon ausgehen, dass es sich bei Erzählungen um autonome Einheiten handelt, widmet sie sich Erzählfragmenten in verschiedenen Gesprächssituationen und Kommunikationsbereichen genauer: Sie geht auf nichtausgebaute, fragmentarische Erzählungen in Gesprächen mit Kindern, in der Arzt-Patienten-Kommunikation, in der Kommunikation zwischen Lehrer und Eltern bei Sprechstundengesprächen ein sowie in sprachbiographischen, narrativen Interviews. Deutlich werden dabei die unterschiedlichen Funktionen fragmentarischen Erzählens. Während es in der Erwachsenen-Kind-Interaktion darum geht, gemeinsam eine vollständige, kohärente Erzählung herzustellen und dem Kind von Seiten der Erwachsenen bei der Herstellung von Kohärenz zu helfen, sind beispielsweise Erzählungen in der ÄrztinPatienten-Kommunikation, in der LehrerIn-Eltern-Interaktion oder in narrativen Interviews häufig nicht vollständig. Sie dienen hier v.a. institutionellen Erfordernissen. 
Die in übergeordnete Aktivitäten eingebetteten Erzählfragmente haben dabei ganz unterschiedliche Funktionen, können beispielsweise der Illustration, der argumentativen Stützung oder der Dramatisierung von Sachverhalten dienen.

Aus einer spracherwerbstheoretischen Perspektive wird der Erwerb der Erzählkompetenz im Beitrag von Uta Quasthoff und Juliane Stude thematisiert. Die Autorinnen zeichnen in ihrem Beitrag »Narrative Interaktion: Entwicklungsaufgabe und Ressource des Erzählerwerbs« verschiedene Erwerbsverläufe narrativer Kompetenz bei Kindern im Grundschulalter mittels einer Längsschnittstudie nach. Ausgangspunkt ihrer Untersuchungen stellt dabei das aus der Wissenssoziologie stammende Konzept der »kommunikativen Gattung « (Luckmann 1988) dar. Erzählungen werden im Anschluss an das Gattungskonzept als ko-konstruierte sprachliche Äußerungspakete verstanden, die in Gespräche eingebettet sind.

Ausgehend von den Erläuterungen der Prinzipien narrativer Interaktion gehen sie darauf ein, wie gemeinschaftlich Erzählungen in der Interaktion zwischen Kindern und Erwachsenen hergestellt werden, wobei sie insbesondere beschreiben, wie die interaktiven Prinzipien als Erwerbsressourcen einerseits und als kontextuell eingebettete Verständigungsressourcen fungieren können. Gegenstand stellen mündlich realisierte, konversationell eingebettete Erlebnis- und Fantasieerzählungen dar. Aus den Daten entwickeln die Autorinnen ein Kompetenzmodell narrativer Kompetenz, das drei Facetten umfasst: die Kontextualisierung, die Vertextung und die Markierung.

Dass mündliche, narrative Alltagspraktiken immer auch verbunden sind mit sichtbar-kinesischen Praktiken zeigen Katharina König und Florence Oloff in ihrem Beitrag »Die Multimodalität alltagspraktischen Erzählens«. Mündliches Erzählen begreifen sie als Praktik, die sich nur aus dem Gebrauchskontext erschließen lässt und zumeist in weitere sprachliche und nicht-sprachliche, körperlich gebundene und verkörperlichte Alltagspraktiken integriert ist. Erzählen als multimodales Geschehen ist dabei sequenziell und immer schon auf den Kontext bezogen, zugleich bringt die Alltagspraktik des Erzählens aber auch Kontext hervor und ist somit beteiligt an der Konstitution von Alltag. Sprachliche und kinesische Praktiken bedingen sich dabei gegenseitig. Ziel des Beitrags ist es, exemplarisch anhand zweier verschiedener Alltagspraktiken des Erzählens die Komplexität des Zusammenspiels multimodaler Ressourcen beim alltäglichen Erzählen zu zeigen, wobei der Beitrag zentrale Erzählphasen - den Einstieg, die Ausgestaltung und den Ausstieg - fokussiert. Deutlich wird bereits bei diesen beiden Beispielen, wie unterschiedlich Erzählungen in der Face-to-face-Interaktion situiert und gerahmt sein können und wie unterschiedlich dabei visuelle Ressourcen, insbesondere körperliche Aktivitäten gestischer Art, zum Einsatz kommen. Ebenso deutlich wird durch den Beitrag gezeigt, dass alltägliches Erzählen in Face-to-face-Interaktionen einen gemeinsamen Prozess der Herstellung von Erzählungen darstellt, bei dem es klare und feste Beteiligungsrollen nicht gibt. Vielmehr übernehmen die Akteure unterschiedliche Rollen wie ZuhörerIn, ErzählerIn, ZuschauerIn.

Ein zweiter Bereich in diesem Themenheft umfasst Beiträge, die sich mit dem Erzählen in der schriftsprachlichen Kommunikation auseinandersetzen; Gegenstand sind hier zum einen literarisch-ästhetische Texte (vgl. Gredel/Mell; Ziem/Lasch), internetbasierte Formen des Erzählens, etwa in sozialen Netzwerken (vgl. Weidacher), 
in Internetforen (vgl. Bubenhofer), in gemeinschaftlich hergestellten Onlineenzyklopädien (Gredel/Mell) oder in Print- und Onlinetageszeitungen (Ziem/Lasch). Die einzelnen Beiträge praktizieren jeweils unterschiedliche Zugriffsweisen auf das zu untersuchende Datenmaterial und es kommen sowohl qualitative als auch quantitative Analysemethoden zum Einsatz. So bieten korpuslinguistische Methoden neue Möglichkeiten, erzählrelevante Muster in größeren Korpora zu entdecken. Dass aus korpuslinguistischer Perspektive die Zugriffe auf das Datenmaterial ausgesprochen unterschiedlich ausfallen können, belegen die Beiträge von Bubenhofer, Gredel/Mell und Ziem/Lasch.

Eine qualitative Studie politischer Kommunikation im Internet liegt mit Georg Weidachers Beitrag »Erzählen als Element politischer Kommunikation in Sozialen Medien« vor. Er zeigt, dass Erzählungen im Kontext politischen Sprachgebrauchs, also im Kontext der Kommunikationsdomäne Politik, eine besondere Rolle für die Perspektivierung von Kommunikation im Sinne der Etablierung politischer Mythen spielt. Dabei wird deutlich, dass Narrationen zur identitätsstiftenden, sozialen Gruppenbildung v. a. auch in den Sozialen Medien genutzt werden. Durch die Analyse wird offensichtlich, dass Narrationen in diesem Kommunikationsbereich unter anderem dem Zweck der politischen Propaganda dienen. Weidacher demonstriert dies am Beispiel des österreichischen Bundespräsidentschaftswahlkampfs.

Ausgehend von der Erörterung des bislang in diskurslinguistischen Zusammenhängen kaum thematisierten Narrativbegriffs befassen sich Eva Gredel und Ruth Mell in ihrem Beitrag »Narrrative - diskursiv und digital: Zum Einsatz digitaler Tools und Ressourcen für Diskursanalysen von Narrativen und von narrativen Konzepten« mit der Frage nach geeigneten korpuslinguistischen Methoden und Instrumenten zur Untersuchung von Narrativen in der digitalen, internetbasierten Kommunikation. In Auseinandersetzung mit wissenssoziologischen Narrativ-Konzepten sprechen sich die Autorinnen für eine Implementierung des Narrativ-Begriffs in die Diskurslinguistik aus. Exemplarisch zeigen sie für das Pflicht-Narrativ literarischer Texte des 19. Jahrhunderts, wie aus einer diskurslexikographischen Perspektive Narrative untersucht werden können, um dann einen Ausschnitt aus dem digital geführten Nanotechnologiediskurs im Hinblick auf Narrative zu analysieren. Die Konzentration bzw. Fokussierung auf einzelne Wörter ist dem diskurslexikographischen Ansatz geschuldet. Dieser geht davon aus, dass einzelne Lexeme als Diskursverdichtungen aufzufassen sind. Denn sie sind immer schon eingebunden in ein kontextuelles bzw. intertextuelles Verweisnetz und können für komplexe Erzählungen und/oder Argumentationen stehen. Auf der Basis einer lexemgebundenen Analyse als diskurslinguistischer Ausgangspunkt können so Narrative aufgespürt werden.

Mit der Etablierung narrativer Sprachgebrauchsmuster in der digitalen Forenkommunikation befasst sich der Beitrag »Serialität der Singularität: Korpusanalyse narrativer Muster in Geburtsberichten« von Noah Bubenhofer. Der Autor fokussiert in seinem Beitrag auf der Datengrundlage eines Korpus von 14.000 Geburtsberichten die Analyse typischer Sprachgebrauchsmuster, die die Narration >Geburtsbericht $<$ in Onlineforen konstituieren. Mit der Analyse der verwendeten Muster soll deutlich gemacht werden, welche Vorstellungen, Handlungs- und Denkmuster im deutschsprachigen Raum (Deutschland, Österreich und Schweiz) das Erzählen von und über Geburten bestimmen. Bubenhofer zeigt, dass die Geburtsberichte sich gut in die 
von Labov/Waletzky (1967) herausgearbeiteten Erzählphasen (Orientierung, Komplikation, Evaluation, Resolution, Koda) einordnen lassen. Innerhalb dieser Phasen dominieren bestimmte Muster, die zunächst als n-Gramme näher beschrieben werden können, schließlich aber interpretativ zu narrativen Toposklassen - Erwartung, Selbstbestimmung, Fremdbestimmung, Egozentrierung, Euphorisierung und Initiation - ordnen lassen, die sich wiederum aus narrativen Topoi speisen.

Ein weiterer linguistischer Zugriff auf narrative Muster alltäglichen Erzählens liegt mit dem Beitrag »Konstruktionsgrammatische Zugänge zu narrativen Texten: Ausgangspunkte und Perspektiven« von Alexander Ziem und Alexander Lasch vor. Die Autoren gehen davon aus, dass alltägliches Erzählen bestimmt ist durch Musterhaftigkeit, die sich auf unterschiedlichen sprachstrukturellen Ebenen und aus unterschiedlichen linguistischen Perspektiven (z. B. interaktional, kognitiv, grammatisch) beschreiben lässt. Dabei gehen sie zunächst von der Alltäglichkeit des Erzählens aus, das sich auf verschiedene Welten beziehen kann (in der Alltagswelt, aber auch in der Welt der Fiktionen) und sich im Luckmann'schen Sinne in kommunikativen Gattungen niederschlägt, die als »routinisierte und mehr oder weniger verpflichtende Lösungen für bestimmte kommunikative Probleme« (Luckmann 1988, S. 282) aufzufassen sind. Die Autoren legen ihren Schwerpunkt gezielt auf die Ebene der grammatischen Konstitution von narrativen Texten und fragen danach, ob es sprachliche Strukturen gibt, die »narrationsspezifische Funktionen übernehmen«. Ausgehend von der These, dass es gattungsspezifische Muster gibt, die in Erzählungen Konstruktionsstatus aufweisen, werden zwei Fallbeispiele von Konstruktionen, die wichtig für narrative Gattungen sind, genauer analysiert. Es handelt sich um den narrativen Konnektor und dann sowie um die nonagentive Konstruktion der Askription mit aussehen.

Danksagung An dieser Stelle möchten wir uns für die gründliche und umsichtige redaktionelle Bearbeitung der Beiträge bei Kristina Stog und Julia Radtke bedanken.

\section{Literatur}

Bal, Mieke: »Interdisciplinary approaches to narrative«. In: David Herman/Manfred Jahn/Marie-Laure Ryan (Hg.): Routledge encyclopedia of narrative theory. London (u. a.) 2007, S. 250-252.

Becker, Tabea: »Erzählentwicklung beschreiben, diagnostizieren und fördern«. In: Carmen Spiegel/ Michael Krelle (Hg.): Sprechen und Kommunizieren in der Schule. Baltmannsweiler 2009, S. 64-81.

Becker, Tabea: »Erzählkompetenz«. In: Matías Martínez (Hg.): Handbuch Erzählliteratur. Stuttgart 2011, S. 58-63.

Becker, Tabea/Stude, Juliane: Erzählen. Kurze Einführung in die Germanistische Linguistik. Heidelberg 2017.

Berger, Peter/Luckmann, Thomas: Die gesellschaftliche Konstruktion der Wirklichkeit. Eine Theorie der Wissenssoziologie. Mit einer Einleitung zur deutschen Ausgabe von Helmuth Plessner. Übersetzt von Monika Plessner. 20. Aufl. Frankfurt 2004.

Birkner, Karin: »Erzählen im Arzt/Patient-Gespräch«. In: Martin Huber/Wolf Schmid (Hg.): Grundthemen der Literaturwissenschaft: Erzählen. Berlin 2017, S. 547-566.

Bubenhofer, Noah/Müller, Nicole/Scharloth, Joachim: »Narrative Muster und Diskursanalyse: Ein datengeleiteter Ansatz«. In: Zeitschrift für Semiotik, Methoden der Diskursanalyse Bd. 35 (2013), Nr. 3-4, S. 419-444.

De Fina, Anna/Georgakopoulou, Alexandra (Hg): Handbook of narrative analysis. New York 2015.

Fix, Ulla: »Zugänge zu Textwelten. Linguistisch-literaturwissenschaftliche Möglichkeiten, in die Geschlossenheit eines Erzähltextes einzudringen«. In: Fritz Hermanns/Werner Holly (Hg.): Lin- 
guistische Hermeneutik. Theorie und Praxis des Verstehens und Interpretierens. Tübingen 2007, S. 323-356.

Fix, Ulla: »Mögliche Zugänge zur Textualität: Wortfelder, Frames, Scripts, Narrationen. Nachtrag zum Textlinguistischen Repetitorium«. In: Urska Valencic Arh/Darko Cuden (Hg.): Im Labyrinth der Sprache. Ljubljana 2015, S. 95-104.

Günthner, Susanne: »Beschwerdeerzählungen als narrative Hyperbeln«. In: Jörg Bergmann/Thomas Luckmann (Hg.): Kommunikative Konstruktion von Moral. Opladen 1999, S. 174-205.

Günthner, Susanne: »Narrative reconstructions of past experiences. Adjustments and modifications in the process of recontextualizing a past experience«. In: Uta Quasthoff/Tabel Becker (Hg.): Narrative Interaction. Amsterdam/New York 2005, S. 285-301.

Günthner, Susanne: »Kleine interaktionale Erzählungen als Ressourcen der Fremd- und Selbststilisierung «. In: Friederike Kern/Miriam Morek/Sören Ohlhus (Hg.): Erzählen als Form - Formen des Erzählens. Berlin/Boston 2012, S. 65-83.

Kern, Friederike/Morek, Miriam/Ohlhus, Sören (Hg.): Erzählen als Form - Formen des Erzählens. Berlin/ Boston 2012.

Kotthoff, Helga: »Besondere Formen des Erzählens in Interaktionen. Vom Klatsch über den Bericht bis zum Witz und spaßigen Phantasien«. In: Stefan Habscheidt (Hg.): Textstrukturen, Kommunikationstypologien, Oberflächen. Berlin 2011, S. 389-413.

Kotthoff, Helga: »Narrative constructions of school-oriented parenthood during parent-teacher-conferences«. In: Linguistics and Education 31 (2015), S. 286-303.

Labov, William/Waletzky, Joshua: »Narrative analysis: Oral versions of personal experience«. In: June Helm (Hg.): Essays on the verbal and visual arts. Seattle 1967, S. 12-44.

Leonardi, Simona/Eva-Maria, Thüne/Anne, Betten (Hg.): Emotionsausdruck und Erzählstrategien in narrativen Interviews. Analysen zu Gesprächsaufnahmen mit jüdischen Emigranten. Würzburg 2016.

Luckmann, Thomas: »Kommunikative Gattungen im kommunikativen Haushalt einer Gesellschaft «. In: Gisela Smolka-Koerdt/Peter M. Spangenberg/Dagmar Tillmann-Bartylla (Hg.): Der Ursprung von Literatur. Medien, Rollen, Kommunikationssituationen zwischen 1450 und 1650. München 1988, S. 279-288.

Mandelbaum, Jenny: »How to do things with narrative. A communication perspective on narrative skill«. In: John O. Greene/Brant Raney Burleson (Hg.): Handbook of communication and social interaction skills. Mahwah 2003, S. 595-633.

Martínez, Matías (Hg.): Erzählen. Ein interdisziplinäres Handbuch. Stuttgart 2017.

Norrick, Neal R.: »Conversational storytelling«. In: David Herman (Hg.): The Cambridge companion to narrative. Cambridge 2007, S. 127-141.

Papenbrock, Martin/Tophinke, Doris: »>Es war eine dieser Nächte ...< Writer Storys zwischen Fiktionalität und Faktualität«. In: Der Deutschunterricht 4 (2016), S. 57-67.

Quasthoff, Uta M.: Erzählen in Gesprächen. Tübingen 1980.

Quasthoff, Uta M.: »Erzählen als interaktive Struktur«. In: Klaus Brinker/Gerd Antos/Wolfgang Heinemann/Sven F. Sager (Hg.): Text- und Gesprächslinguistik. Ein internationales Handbuch zeitgenössischer Forschung. Berlin/New York 2001, S. 1293-1309.

Rehbein, Jochen: »Homileïscher Diskurs - Zusammenkommen, um zu reden«. In: Friederike Kern/ Miriam Morek/Sören Ohlhus (Hg.): Erzählen als Form - Formen des Erzählens. Berlin/Boston 2012, S. 85-108.

Schegloff, Emanuel A.: »>Narrative analysis< thirty years later«. In: Journal of narrative and life history 7:1-4 (1997), S. 97-106.

Tophinke, Doris: »Lebensgeschichte und Sprache. Zum Konzept der Sprachbiografie aus linguistischer Sicht«. In: Bulletin suisse de linguistique appliquée 76 (2002), S. 1-14.

Tophinke, Doris: »Wirklichkeitserzählungen im Internet«. In: Christian Klein/Matías Martínez (Hg.): Wirklichkeitserzählungen. Felder, Formen und Funktionen des nicht-literarischen Erzählens. Weimar 2009, S. 245-274.

Tophinke, Doris: »Erzählen im Internet«. In: Matías Martínez (Hg.): Erzählen. Ein interdisziplinäres Handbuch. Stuttgart 2017, S. 70-75. 